\title{
A Capacitação dos Utentes Quanto aos Rastreios Médicos
}

\section{The Empowerment of Health Care Unit Users Regarding Medical Screenings}

Filipa Sousa*, Luiz Miguel Santiago**, Vitor Rodrigues ${ }^{* * *}$

\section{Pontos-chave:}

A prevenção em saúde é importante mas é amiúde excessivamente usada.

A crença populacional e o conhecimento sobre rastreios que os nossos consulentes têm.

Sendo grande a aceitação da realização de rastreios sobre doenças, urge perceber como melhor informar sobre rastreios pois estes não permitem diagnosticar doença nem podem servir para dar a falsa sensação de estar saudável nem para induzir o medo de doença.

\section{Resumo}

Objectivos: Avaliar a capacitação de utilizadores de unidades de saúde quanto à realização de rastreios médicos.

Tipo de estudo: Observacional.

Local: Unidades de Saúde de Cuidados de Saúde Primários do concelho de Viseu (USF Grão Vasco, USF Viseu Cidade, USF Lusitana e UCSP D. Duarte).

População: Utentes inscritos nas quatro unidades de saúde.

Métodos: Aplicação de um questionário a uma amostra representativa, de 400 indivíduos, por sorteio das unidades de saúde onde decorreu o estudo, entre Julho e Agosto de 2014. As respostas obtidas foram estudadas em função da percepção da qualidade de saúde e de variáveis sociodemográficas. Análise inferencial através da ferramenta IBM SPSS Statistics.

Resultados: A amostra tem idades compreendidas entre os 18 e os 89 anos, média de 51 anos. Dos inquiridos, $93,8 \%$ aceitam a realização de um rastreio médico sempre que lhe é proposto e $87,8 \%$ julgam que essa participação permite saber que têm uma doença. Há maior

\section{Abstract}

Objectives: Evaluate the capacity development of health care unit users insofar as to medical screenings.

Type of study: Observational.

Setting: Unidades de Saúde de Cuidados de Saúde Primários do concelho de Viseu (USF Grão Vasco, USF Viseu Cidade, USF Lusitana e UCSP D. Duarte).

Population: Patients registered in the four health units.

Methods: Use of a questionnaire on a representative sample of 400 individuals, through the draw of health units where the study was held, between July and August of 2014. The responses obtained were studied taking into account the perception of quality of health and socio-demographic factors. Inferential analysis by IBM SPSS Statistics.

Results: The sample comprises individuals between the age of 18 and 89, average age 51. The respondents accept a medical screening whenever one is proposed to them in $93,8 \%$ and $87,8 \%$ believe that taking part in them enables them to know if they have a disease. There is greater acceptance of medical screenings amongst

\footnotetext{
*Aluna do $6^{\circ}$ ano médico da Faculdade de Medicina da Universidade de Coimbra

${ }^{* *}$ MD, Assistente Graduado Sénior, USF Topázio, ACES Baixo Mondego; PhD, Professor Associado da Faculdade de Ciências da Saúde da Universidade da Beira Interior.

${ }^{* * *}$ MD, Professor Associado da Faculdade de Medicina da Universidade de Coimbra
} 
aceitação dos rastreios médicos no género feminino $(95,6 \%)$ e nos que qualificam a sua saúde como "razoável ou fraca” (96,6\%). Nos inquiridos com grau de formação académica elevado há menor aceitação (11,8\%). À questão "Julga que fazer um rastreio permite saber que tem uma doença?" os utentes da UCSP respondem negativamente numa percentagem mais elevada (16,9\%). Existe diferença significativa entre as motivações de aceitação dos rastreios médicos e as variáveis grupo etário, toma regular de medicamentos, grau de formação académica e qualidade em saúde ( $p<0,001)$.

Conclusões: Há uma grande aceitação dos rastreios médicos. A maioria dos inquiridos julga que fazer um rastreio permite saber que tem uma doença. A realização de rastreios com o intuito de querer saber que está saudável salienta a falsa sensação de protecção e garantia de saúde perante um resultado falso negativo.

Palavras-chave: "Prevenção Secundária”, "Rastreios Médicos”, “Prevenção Quaternária”, “Disease Mongering”, "Literacia em Saúde"

\section{Introdução}

Numa época em que o conhecimento e as novas tecnologias aumentam rapidamente, a prevenção da doença baseada em rastreios médicos é uma das áreas da Medicina Preventiva que mais cresceu nas últimas décadas.

A popularidade criada em torno do conceito de prevenção em saúde tem conduzido a expectativas irrealistas relativamente a alguns dos cuidados preventivos, nos quais a relação benefício/risco não coincide com o que inicialmente se esperava. 0 rastrear de pessoas assintomáticas, em grupos populacionais progressivamente maiores e com limiares de anormalidade menores, ${ }^{1}$ assim como a inclusão de factores de risco no conceito de prevenção, ${ }^{2}$ culmina em opiniões divergentes a favor e contra os rastreios médicos.

Todos os cuidados médicos têm o potencial de provocar dano ao doente e, os preventivos, não são excepção. ${ }^{3}$ As condicionantes éticas no caso dos rastreios são preponderantes, pois os riscos de prejuízo não estão contrabalançados com um risco real mas sim, com um potencial futuro de doença ou morte. ${ }^{4}$ De facto, todos os efeitos adversos dos rastreios médicos são iatrogénicos e passíveis de ser evitados. ${ }^{5}$

No actual período de contenção de custos, a preocupação com a utilização indevida dos recursos é imprescindível e females (95,6\%) and amongst those that qualify their health as "reasonable or poor" (96,6\%). Respondents with higher education levels are less accepting (11,8\%) of screenings. As to the question "Do you believe that a screening allows detecting a disease?", a higher percentage of UCSP users provided a negative response (16,9\%). There is a significant difference as to the motivations for accepting medical screenings among the age group, regular medication-taking, education level, and quality of health variables $(p<0,001)$.

Conclusions: Medical screenings are widely accepted. The majority of respondents feel that medical screenings enable detecting a disease. Screenings carried out for the purpose of wanting to know one is healthy provide a false sensation of protection and guarantee of health given a false negative result.

Key words: "Secondary Prevention", "Medical Screenings", "Quaternary Prevention", "Disease Mongering”, "Health Literacy"

os programas de rastreios não se excluem desta temática. ${ }^{6}$ Contudo, os custos raramente são a razão pela qual as guidelines limitam o uso de determinados rastreios. A maior parte da controvérsia surge com o paradigma da relação entre benefício e dano. ${ }^{7}$

Uma revisão sistemática publicada no British Medical Journal afirma que apenas 30\% dos ensaios clínicos controlados e aleatorizados se preocupam em avaliar os possíveis danos dos rastreios. ${ }^{4}$ Este dano potencial pode ocorrer na sequência de complicações do próprio teste, da possibilidade de falsos e verdadeiros positivos e ainda de malefícios provocados pela cascata diagnóstica e terapêutica de um falso positivo, frequentemente envolvendo procedimentos invasivos e dispendiosos. ${ }^{8}$

Um extremo de viés, reconhecido como um dos principais riscos dos rastreios, é o designado sobrediagnóstico, ${ }^{1}$ em que a doença detectada nunca irá ser clinicamente significativa ou causar a morte. ${ }^{9}$

O rastreio do cancro da próstata com o teste PSA contribui para o aumento da sua incidência no final dos anos 80. ${ }^{10,11}$ Este tipo de cancro é possivelmente o que apresenta maior risco de sobrediagnóstico, estimado por Brawley em 50-70\%.12 Apesar do comportamento indolente desses tumores, 90\% são tratados com radiação 
ou cirurgia o que acarreta morbilidade como incontinência urinária, disfunção sexual e efeitos gastrointestinais, em cerca de $15-20 \%$ dos casos. ${ }^{11,13}$

Num estudo realizado por Wegwarth e Gigerenz, a maioria dos participantes de rastreios oncológicos de rotina refere não ter sido informado acerca do sobrediagnóstico e subsequente tratamento desnecessário. Cerca de 69\% afirma não iniciar o rastreio se o sobrediagnóstico for tão elevado como é nos casos da mamografia e teste PSA. ${ }^{9}$

Apesar do aumento do conhecimento médico, algumas decisões na prática clínica são ainda pautadas pela incerteza $^{3}$ e, frequentemente, o médico é outorgado pelo doente acerca de uma convicção que não possui. ${ }^{2}$

Segundo Jamoulle, a prática da medicina clínica resulta do equilíbrio entre a "gestão da incerteza" diagnóstica e a limitação dos riscos terapêuticos. ${ }^{14}$

Desde 1997, com a declaração de Jacarta, que existe uma preocupação crescente relativamente à sobremedicalização. ${ }^{15}$ É neste contexto que Marc Jamoulle propõe em 1995, no encontro do Comitê Internacional de Classificação da WONCA, um novo nível de prevenção em saúde, a prevenção quaternária.16 Este conceito é aceite pelo Comitê em 1999, que publica em 2003, no WONCA Dictionary for General/Family Practice, ${ }^{17}$ a definição de prevenção quaternária - "acção desenvolvida para identificar doentes em risco de sobremedicalização, para os proteger de nova intervenção médica inapropriada e sugerir-Ihes alternativas eticamente aceitáveis".,18

Este quarto e último tipo de prevenção é igualmente designado "prevenção da iatrogenia" ou "prevenção da prevenção" e tenta recuperar um dos fundamentos centrais da medicina, o preceito hipocrático primum non nocere. ${ }^{19,20}$ Segundo Gérvas, tem como objectivo não só evitar ou atenuar o intervencionismo médico ${ }^{21}$ e a medicalização desnecessária 22 mas, também, capacitar os utentes para a tomada de decisões autónomas, fornecendo-Ihes as informações necessárias acerca das vantagens e desvantagens das intervenções propostas. ${ }^{21}$

Para Kuehlein et al., a prevenção quaternária enquadra o problema da enfermidade sem doença. ${ }^{17}$ Trata-se de uma exigência constante e omnipresente em toda a prática clínica, que engloba todo o tipo de actividades médicas sejam diagnósticas, terapêuticas, preventivas ou curativas. ${ }^{23}$

Adicionalmente, estamos perante um paradoxo de uma sociedade com maior esperança de vida e melhores ín- dices da sua qualidade, mas que está cada vez mais “doente" e mais medicada. ${ }^{3}$ Por conseguinte, surge uma população cada vez mais dependente do sistema de cuidados de saúde.

Consequente ao aparecimento de uma medicina que oferece programas preventivos nem sempre apoiados por evidência científica e à criação de uma opinião pública em que tudo é evitável e curável, surge uma população com reivindicações, em saúde, ilimitadas. ${ }^{24}$ Frequentemente o excesso de intervencionismo resulta da pressão exercida pelos utentes sobre os clínicos, a designada "medicina defensiva". ${ }^{25}$

Apesar de existir um contínuo entre saúde e enfermidade ${ }^{17}$ a tradição médica é de dicotomizá-lo em normal ou anormal, o que torna a definição de doença arbitrária. Neste contexto, surge o interesse da indústria farmacêutica para modificar os limites dessa distinção. ${ }^{26}$

Neste âmbito, surge por Lynn Payer ${ }^{24}$ o conceito de "disease mongering”, no qual são utilizadas estratégias de marketing que sobrestimam situações clínicas ou factores de risco e medicalizam condições fisiológicas, transformando indivíduos saudáveis em "doentes" com fins lucrativos. ${ }^{3}$ Esta concepção de promoção da doença explora o medo do sofrimento e da morte inerentes à condição humana. ${ }^{26}$

A medicalização inapropriada acarreta prejuízos como a criação de rótulos desnecessários, decisões terapêuticas desadequadas, iatrogenia e desperdícios económicos que poderiam ser canalizados para pesquisas na prevenção ou terapêuticas de outras doenças graves. ${ }^{27}$

Desde a década de 50, a Classificação Internacional das Doenças, desenvolvida pela OMS, aumentou notavelmente de 300 itens para cerca de 15000. Relativamente à saúde mental, a classificação mais popular é a DSM, que conta com mais de 350 "condições" na sua quinta e última revisão, sendo já a timidez considerada doença. ${ }^{2}$ A saúde mental é uma das áreas mais susceptiveis ao disease mongering. ${ }^{28}$ De facto, alguns autores defendem que a maioria das doenças constantes na DSM foi incluída pela influência da indústria farmacêutica. ${ }^{2}$

A prevenção quaternária exige autonomia, independência, conhecimento científico sólido e capacidade de comunicação. Muitas vezes é o simples "esperar para ver" numa vertente de atitude expectante. ${ }^{29}$ Assim, deve ser desenvolvida continuamente e em simultâneo com a actividade clínica ${ }^{30}$, procurando o máximo de qualidade com o mínimo de quantidade/intervenção possível. ${ }^{31}$ 
Jamoulle refere dois meios que pensa serem fundamentais para a aplicação da prevenção quaternária. Primeiro, o conhecimento e autodomínio próprios do médico que deve aceitar a sua incompletude e dar primazia a uma boa relação médico-doente, comunicação e empatia. Segundo, o conhecimento e a ciência baseadas em provas sempre que possivel. ${ }^{2}$

A medicina baseada na evidência, originalmente desenvolvida por Sackett e seus colaboradores, representa um dos métodos preponderantes para avaliar processos médicos desnecessários. ${ }^{17}$

Segundo Melo, são igualmente condições favorecedoras deste nível de prevenção, a adopção de uma perspectiva biopsicossocial, aceitação da existência de sintomas clinicamente não explicáveis, actualização constante dos conhecimentos e utilização de protocolos desenvolvidos interpares, adaptados localmente. ${ }^{3,32}$

Outro dos caminhos defendidos é o da capacitação das populações para as decisões em saúde. ${ }^{19}$ Para tal, o doente tem de compreender a doença ou condição para o qual é rastreado e conhecer os benefícios, riscos, limitações, alternativas e incertezas que os serviços médicos envolvem. Associadamente, tem de tomar decisões consistentes com os seus valores e preferências. $^{33,34}$

Já em 1998, a OMS define literacia em saúde como "competências cognitivas e sociais que determinam a capacidade dos indivíduos para obter acesso à compreensão e utilização da informação, de forma a promover e manter uma boa saúde". ${ }^{35}$

Todavia, a noção da capacitação dos utentes para a tomada de decisões informadas levanta alguns problemas. A ciência pode ser difícil de comunicar por parte do médico e difícil de compreender pelo doente. ${ }^{7} 0$ tempo para essa discussão pode ser escasso e existem doentes que depositam no médico a capacidade de decisão. ${ }^{36}$

Da mesma forma, alguns doentes expressam a sua preferência relativamente a determinada intervenção com a qual o médico acaba por concordar, mesmo acreditando ser desnecessária. Uma das razões apontadas é a evicção do confronto e prejuízo da relação médico-doente. ${ }^{36,37}$ Por outro lado, as pressões sociais e médico-legais podem influenciar essa deliberação. ${ }^{7}$

A medicina moderna tem colocado um desafio na relação médico-doente. 0 aspecto hipocrático da medicina com foco no doente tem sido substituído por estratégias de alvo populacional, orientadas para a comunidade. ${ }^{38}$
É necessário compreender os limites do conhecimento médico, praticar menos extrapolação dos resultados de investigação e utilizar a estatística de forma mais responsável. Só assim, a verdadeira relação entre os benefícios e os riscos pode ser correctamente compreendida. ${ }^{26}$

O desafio passa por individualizar cada caso e agir no meio de incertezas, de forma a garantir a informação dos doentes quanto aos potenciais danos e promover decisões em saúde fundamentadas. É neste âmbito que a prevenção secundária se cruza com a prática baseada no novo nível de prevenção, a prevenção quaternária.

Neste contexto e pela escassez de investigação nesta vertente, o presente estudo visa avaliar o conhecimento dos utentes quanto ao conceito de rastrear e conhecer o grau de aceitação e motivação para a participação dos mesmos em programas de rastreio.

\section{Métodos}

Para a realização do estudo foi escolhida uma amostra representativa da população, considerando um intervalo de confiança de $95 \%$ e margem de erro de 5\%, com base no número total de utentes inscritos nas Unidades de Cuidados de Saúde Primários, do concelho de Viseu. As Unidades envolvidas na investigação foram seleccionadas através de sorteio, baseado numa lista das mesmas. 0 tamanho da amostra foi calculado pelo instrumento web Lenth, R. V. (2006-9). Java Applets for Power and Sample Size [Computer software]. Acedido em Março de 2014, no sítio http://www.stat.uiowa. edu/ rlenth/Power, sendo necessária uma amostragem total mínima de 382 inquiridos.

0 instrumento utilizado foi um questionário previamente aplicado num estudo realizado em Coimbra, no âmbito de Tese de Mestrado Integrado em Medicina da Faculdade de Medicina da Universidade de Coimbra, no ano de 2013. Foi elaborado por dois sociólogos, dois psicólogos, três médicos, duas enfermeiras e três pessoas não relacionadas com a área da saúde e validado com um pré-teste a 15 elementos de uma Unidade de Cuidados de Saúde Primários.

O questionário é constituído por uma página com 11 perguntas, sendo cinco referentes às características sócio-demográficas e uma relativa à percepção da qualidade de saúde. As questões relativas aos rastreios médicos englobam as seguintes temáticas "aceitação de rastreios médicos”, "capacidade do rastreio permitir saber que tem a doença" e as premissas de que "mesmo que faça o rastreio pode vir a sofrer de uma doença" 
e "mesmo que faça um rastreio pode vir a morrer de uma doença". Estes campos têm duas opções de preenchimento, "sim" ou "não". Quanto à última pergunta, relativa aos motivos de aceitação dos rastreios médicos, as opções de resposta são "saber que está saudável", "confia nos rastreios", "saber que tem doença" e "aceita as consequências de os fazer".

A aplicação do questionário decorreu nos meses de Julho e Agosto de 2014, a utentes que nesse período se encontravam nas respectivas Unidades de Saúde a aguardar consulta médica ou de enfermagem, com uma amostragem final de 400 indivíduos. Outro critério de inclusão foi a autorização de participação no estudo, com base na assinatura do consentimento informado anexo ao questionário. São critérios de exclusão o analfabetismo e utentes com dificuldade de compreensão dos conceitos envolvidos no questionário e incapacidade de manifestar opinião.

Neste estudo foram utilizadas as seguintes variáveis: idade, variável quantitativa discreta; grupo etário, grau de formação académica e qualidade de saúde, variáveis qualitativas ordinais; género, toma regular de medicamentos, grupo de actividade profissional e grupo de Unidade da Saúde (USF/UCSP), variáveis qualitativas nominais.

Para o tratamento dos dados foi utilizada a ferramenta informática “IBM SPSS Statistics - version 22.0". Na análise inferencial foi utilizado o teste $\chi 2$ para as variáveis nominais e efectuados testes não paramétricos, MannWhitney e Kruskal-Wallis, para as variáveis ordinais. Foi considerado o nível de significância de 0,05.

\section{Resultados}

A caracterização da amostra relativamente às variáveis Unidade de Saúde, grupo etário, género, toma regular de medicamentos, grau de formação académica, actividade profissional e qualidade de saúde é exposta na tabela I.

Tabela I: Caracterização da amostra

\begin{tabular}{|c|c|c|c|}
\hline Variável & $\mathbf{N}^{0}$ & $\%$ & Total $\mathbf{N}^{\circ}(\%)$ \\
\hline \multicolumn{4}{|l|}{ Unidade de Saúde } \\
\hline USF Viseu Cidade & 85 & 21,3 & \multirow{4}{*}{$400(100)$} \\
\hline USF Lusitana & 100 & 25,0 & \\
\hline USF Grão Vasco & 85 & 21,3 & \\
\hline UCSP D. Duarte & 130 & 32,5 & \\
\hline \multicolumn{4}{|c|}{ Grupo de Unidade de Saúde } \\
\hline USF & 270 & 67,5 & \multirow{2}{*}{$400(100)$} \\
\hline UCSP & 130 & 32,5 & \\
\hline \multicolumn{4}{|l|}{ Grupo Etário } \\
\hline$\leq 35$ anos & 71 & 17,8 & \multirow{3}{*}{$400(100)$} \\
\hline $35-65$ anos & 247 & 61,8 & \\
\hline$\geq 65$ anos & 82 & 20,5 & \\
\hline \multicolumn{4}{|l|}{ Género } \\
\hline Feminino & 270 & 67,5 & \multirow{2}{*}{$400(100)$} \\
\hline Masculino & 130 & 32,5 & \\
\hline \multicolumn{4}{|c|}{ Toma regular de medicamentos } \\
\hline Sim & 259 & 64,8 & \multirow{2}{*}{$400(100)$} \\
\hline Não & 141 & 35,3 & \\
\hline \multicolumn{4}{|c|}{ Grau de formação académica } \\
\hline Baixo & 34 & 8,5 & \multirow{3}{*}{$400(100)$} \\
\hline Médio & 273 & 68,3 & \\
\hline Alto & 93 & 23,3 & \\
\hline
\end{tabular}




\begin{tabular}{lccc}
\hline Atividade Profissional & & & \\
\hline Agricultura & 6 & 1,5 & \\
Comércio & 31 & 7,8 & \\
Indústria & 39 & 9,8 & \\
Serviços & 107 & 26,8 & 400 (100) \\
Doméstica & 65 & 16,3 & \\
Desempregrado & 43 & 10,8 & \\
Reformado & 96 & 24,0 & \\
Estudante & 13 & 3,3 & \\
\hline Grupo de actividade profissional & & & \\
\hline Ativo & 248 & 62,0 & \\
Não Ativo & 152 & 38,0 & \\
\hline Qualidade de Saúde & & & \\
\hline Ótima/Muito Boa & 62 & $100)$ \\
Boa & 132 & 33,0 & \\
Razoável/ Fraca & 206 & 51,5 & \\
& & & \\
\hline
\end{tabular}

Não se verifica existir diferença estatisticamente significativa entre os grupos USF e UCSP quanto às variáveis sócio-demográficas e percepção da qualidade em saúde.
Na tabela II, apresentam-se os resultados obtidos relativamente às questões referentes aos rastreios médicos em função da amostra total.

Tabela II: Resposta às questões em função da amostra total

\begin{tabular}{|c|c|c|}
\hline Questão & $\mathbf{N}^{\circ}$ & $\%$ \\
\hline \multicolumn{3}{|c|}{ Sempre que Ihe propõem um "rastreio" em saúde aceita fazê-lo? } \\
\hline Sim & 375 & 93,8 \\
\hline Não & 25 & 6,3 \\
\hline \multicolumn{3}{|c|}{ Julga que fazer um "rastreio" permite saber que tem uma doença? } \\
\hline Sim & 351 & 87,8 \\
\hline Não & 49 & 12,3 \\
\hline \multicolumn{3}{|c|}{ Mesmo que faça um "rastreio" pode vir a sofrer de uma doença? } \\
\hline Sim & 342 & 85,5 \\
\hline Não & 58 & 14,5 \\
\hline \multicolumn{3}{|c|}{ Mesmo que faça um rastreio pode vir a morrer de uma doença? } \\
\hline Sim & 356 & 89,0 \\
\hline Não & 44 & 11,0 \\
\hline \multicolumn{3}{|c|}{ Sabendo do seu estado de saúde por qual ou quais das razões abaixo aceita fazer "rastreios"? } \\
\hline Quer saber que está saudável & 276 & 69,2 \\
\hline Confia nos rastreios & 204 & 51,2 \\
\hline Quer saber que tem doença & 187 & 47,0 \\
\hline Aceita as consequências de os fazer & 183 & 45,9 \\
\hline
\end{tabular}


No que concerne à questão "Sempre que Ihe propõe um rastreio em saúde aceita fazê-lo?" existe diferença estatisticamente significativa em relação à variável género $(p=0,030)$, grau de formação académica $(p=0,038)$ e qualidade em saúde $(p=0,006)$. É de notar que existe uma maior aceitação dos rastreios médicos pelo género feminino $95,6 \%(n=258)$ em contraponto a $90,0 \%(n=117)$ do género masculino. Adicionalmente, os indivíduos com grau de formação académica elevado são também os menos receptivos à realização dos rastreios médicos, contando com uma percentagem de $11,8 \%(n=11)$. Os inquiridos que qualificam a sua saúde como "razoável/fraca" aceitam mais frequentemente a realização de rastreios médicos [96,6\% $(n=199)$ ] em oposição ao grupo dos indivíduos com uma saúde "óptima/muito boa" em que existe uma tendência para uma menor aceitação [14,5\% $(n=9)]$.

Quanto à questão "Julga que fazer um rastreio permite saber que tem uma doença?" apenas se verifica diferença estatisticamente significativa no que diz respeito à variável "Unidade de Saúde" ( $p=0,048)$. Os utentes de USF revelam uma percentagem superior de respostas afirmativas [90,0\% $(n=243)]$, enquanto os inquiridos na UCSP respondem negativamente numa percentagem mais elevada $[16,9 \%(n=22)]$.

No que concerne à questão "Mesmo que faça um rastreio pode vir a sofrer de uma doença?" evidencia-se diferença estatisticamente significativa quanto à variável "toma regular de medicamentos" $(p=0,019)$. É de notar que o grupo que toma regularmente medicamentos apresenta maior percentagem de respostas afirmativas comparativamente ao grupo que não toma [88,4\% ( $n=229)$ vs 80,1\% $(n=113)]$. Assim, o grupo que não toma medicação regular apresenta maior proporção de utentes que acreditam que com a realização do rastreio médico não vão sofrer de uma doença [19,9\% $(n=28)]$.

De igual forma, na questão "Mesmo que faça um rastreio pode vir a morrer de uma doença?" observa-se diferença estatisticamente significativa na inferenciação relativa à variável "toma regular de medicamentos" $(p=0,049)$. Verifica-se que quem toma medicamentos regularmente responde mais frequentemente "sim" [91,1\% (n=236)] e quem não toma contrapõe negativamente em maior percentagem [14,9\% $(n=21)]$.

Quanto à questão relativa às motivações de aceitação dos rastreios médicos existe diferença estatisticamen- te significativa quanto às variáveis grupo etário, toma regular de medicamentos, grau de formação académica e qualidade em saúde ( $p<0,001)$.

Nos grupos etários mais jovens, “< 35 anos" e "35-65 anos", existe uma maior percentagem de indivíduos que "quer saber que está saudável", 70,3\% (n=50) e 69,2\% $(n=171)$, respectivamente. Enquanto isso, no grupo etário acima dos 65 anos, a maioria dos utentes refere como motivação para a realização de rastreios médicos o facto de "querer saber que tem a doença" [73,1\% $(n=60)]$.

Comparativamente aos indivíduos que tomam medicação regularmente, aqueles que negam o seu consumo apresentam um valor percentual inferior no que respeita às seguintes motivações: "quer saber que está saudável" $[70,7 \%(n=183)$ vs $65,8 \%(n=93)]$; "confia nos rastreios" [59,1\% ( $n=153)$ vs 36,0\% ( $n=51)]$; "quer saber que tem doença" [56,3\% ( $n=146)$ vs $29,0 \%(n=41)]$ e "aceita as consequências de os fazer" [57,5\% ( $n=149)$ vs $24,0 \%(n=34)]$.

No que diz respeito ao grau de formação académica verifica-se que os indivíduos de baixo grau apresentam como resposta mais frequente a aceitação das consequências dos rastreios médicos, $76,3 \%(n=26)$, enquanto os indivíduos de médio e elevado grau referem como principal motivação para a participação nos rastreios médicos o facto de desejarem saber que estão saudáveis, 70,7\% $(n=193)$ e $64,5 \%(n=60)$, respectivamente.

Quanto à qualidade em saúde constata-se que os utentes que consideram a sua saúde "razoável/fraca" apresentam as quatro motivações numa percentagem superior aos restantes grupos: "quer saber que está saudável", $70,4 \%$ ( $n=145)$; “confia nos rastreios”, (62,0\% n=128); "quer saber que tem a doença", 55,8\% (n=115) e "aceita as consequências de os fazer", 59,7\% ( $n=123)$.

Como resultado da análise inferencial não se observa diferença estatisticamente significativa em relação a qualquer outra variável.

A tabela III apresenta os resultados da análise comparativa entre a população do estudo realizado em Viseu e uma outra estudada em Coimbra, em 2013, e os respectivos valores de $p$. 0 estudo realizado em Coimbra pretendeu estudar a importância atribuída pelos utentes aos rastreios médicos e a sua amostra foi obtida a partir de uma lista de Unidades de Saúde do concelho de Coimbra, mediante sorteio após ordenação alfabética. 
Tabela III: Análise comparativa entre duas populações

\begin{tabular}{|c|c|c|c|}
\hline \multirow[t]{2}{*}{ Variável } & \multicolumn{2}{|c|}{ Concelho } & \multirow[t]{2}{*}{$p$} \\
\hline & Viseu $\mathrm{N}^{\circ}(\%)$ & Coimbra $\mathrm{N}^{\circ}(\%)$ & \\
\hline \multicolumn{4}{|l|}{ Toma regular de medicamentos } \\
\hline Sim & $259(64,8)$ & $288(72,0)$ & \multirow{2}{*}{0,017} \\
\hline Não & $141(35,3)$ & $112(28,0)$ & \\
\hline \multicolumn{4}{|l|}{ Grupo de actividade profissional } \\
\hline Ativo & $248(62,0)$ & $209(52,3)$ & \multirow{2}{*}{0,003} \\
\hline Não Ativo & $152(38,0)$ & $191(47,8)$ & \\
\hline \multicolumn{4}{|l|}{ Qualidade de Saúde } \\
\hline Ótima/Muito Boa & $62(15,5)$ & $55(13,8)$ & \multirow{3}{*}{0,015} \\
\hline Boa & $132(33,0)$ & $101(25,3)$ & \\
\hline Razoável/ Fraca & $206(51,5)$ & $244(61,0)$ & \\
\hline \multicolumn{4}{|l|}{ Unidade de Saúde } \\
\hline USF & $270(67,5)$ & $201(50,2)$ & \multirow{2}{*}{$<0,001$} \\
\hline UCSP & $130(32,5)$ & $199(49,8)$ & \\
\hline \multicolumn{4}{|c|}{ Julga que fazer um rastreio permite saber que tem uma doença? } \\
\hline Sim & $351(87,8)$ & $373(93,3)$ & \multirow{2}{*}{0,005} \\
\hline Não & $49(12,3)$ & $27(6,8)$ & \\
\hline \multicolumn{4}{|c|}{ Sabendo do seu estado de saúde por qual ou quais das razões abaixo aceita fazer "rastreios"? } \\
\hline Quer saber que está saudável & $276(69,2)$ & $173(43,5)$ & \multirow{4}{*}{$<0,001$} \\
\hline Confia nos rastreios & $204(51,2)$ & $144(36,2)$ & \\
\hline Quer saber que tem doença & $187(47,0)$ & $113(28,5)$ & \\
\hline Aceita as consequências de os fazer & $183(45,9)$ & $94(23,7)$ & \\
\hline
\end{tabular}

\section{Discussão}

Com este estudo denota-se que existe uma grande aceitação dos rastreios médicos [93,8\% $(n=375)]$.

Quanto aos indivíduos que consideram a sua saúde "razoável/fraca" a maior tendência que evidenciam na aceitação da participação em rastreios médicos pode estar ligada ao facto de se encontrarem numa fase de maior fragilidade ou pela possibilidade de uma maior utilização dos cuidados de saúde. Num estudo realizado por Nguyen e seus colaboradores, os indivíduos que utilizam os cuidados de saúde de forma mais regular apresentam uma probabilidade três vezes superior de adesão aos rastreios. ${ }^{39}$

Este resultado corrobora um estudo realizado por Cooper e seus colaboradores, no qual a adesão ao rastreio diminui com um melhor estado de saúde. ${ }^{40}$
Igualmente, o género feminino é aquele que mais frequentemente quer e consente a realização de rastreios médicos. Factores culturais ou sociais podem estar associados a este resultado bem como o sucesso da implementação de programas de rastreio médicos dirigidos exclusivamente ao género feminino. São exemplos, o rastreio do cancro da mama e do colo do útero, que se encontram amplamente instituídos na região centro do país. Para Odgen, a forma como os Serviços de Saúde estão estruturados pode ter implicações no grau de adesão, que é facilitada se existir uma boa acessibilidade. ${ }^{41}$

Associadamente, as mulheres recorrem mais aos serviços de cuidados de saúde primários que os homens ${ }^{42}$, o que pode motivar esta diferença de género, tendo por base o estudo de Nguyen acima referido. 
No que concerne ao grau de formação académica verifica-se que o grupo com formação mais elevada aceita menos frequentemente a realização de rastreios médicos. Esta situação pode estar relacionada com o facto de este grupo consentir numa percentagem inferior as consequências dessa participação. Neste contexto, será importante em estudos futuros avaliar quais os conhecimentos dos utentes relativamente às consequências que a realização de um rastreio implica.

Apesar de não existirem diferenças estatisticamente significativas entre os inquiridos pertencentes a USF ou UCSP, verifica-se que existe diferença entre a variável "Unidade de saúde" e a questão "Julga que fazer um rastreio permite saber que tem uma doença?”. Os utentes da UCSP respondem negativamente numa percentagem mais elevada, o que pode ser justificado pela possibilidade de desigualdade no conhecimento dos fundamentos e objectivos dos rastreios médicos. Este resultado pode ainda ser condicionado por uma actividade de oferta de rastreios diferente entre as duas unidades de saúde. Já em Coimbra, resultado similar foi encontrado, o que indicia uma necessidade de avaliar possíveis diferenças na oferta de rastreios médicos e na literacia em saúde, em função da tipologia de Unidade de Saúde.

Surpreendentemente, $87,8 \%(n=351)$ dos inquiridos julga que fazer um rastreio permite saber que tem uma doença, o que demonstra a presença de uma crença errada acerca da capacidade diagnóstica deste método de prevenção secundária e um défice na literacia em saúde. Visto existir uma grande aceitação dos rastreios médicos, este resultado evidencia que a participação nos mesmos recai na tomada de decisões em saúde não fundamentadas.

O grupo dos utentes que toma medicamentos regularmente apresenta uma percentagem superior de indivíduos que sabem que o rastreio não elimina a possibilidade de vir a sofrer ou morrer por uma doença. Habitualmente, os utentes que consomem regularmente medicamentos recorrem com mais frequência aos cuidados de saúde. Neste âmbito, existe a possibilidade de uma relação médico-doente de maior proximidade e continuidade, que favoreça o conhecimento e o esclarecimento de dúvidas relativas aos rastreios médicos. De facto, é na relação médico-doente que radica a possibilidade de uma efectiva prevenção quaternária e de promoção da capacitação dos utentes.

Relativamente às motivações de participação nos rastreios médicos evidenciam-se como principais as seguintes: "querer saber que está saudável", 69,2\% $(n=276)$ e "confia nos rastreios", 51,2\% $(n=204)$. 0 facto de os utentes realizarem rastreios com o intuito de querer saber que estão saudáveis, destaca a necessidade de considerar um dos riscos dos rastreios, a falsa sensação de protecção e garantia de saúde perante um resultado falso negativo.

Os mais jovens mencionam como principal motivação para a realização dos rastreios o desejo de saber que estão saudáveis. Contrariamente, os mais idosos referem numa percentagem superior a razão "querer saber que tem a doença”. Esta diferença pode deverse ao maior risco de deterioração do estado de saúde neste grupo etário, com o aparecimento de patologias inerentes à condição do envelhecimento e ainda, de uma separação cada vez mais ténue entre o conceito de saúde e doença. É neste contexto de procura pelo conhecimento da doença e de medo de morrer, sobretudo nos mais idosos, que assume particular destaque o conceito de "disease mongering".

A presença de uma diferença estatisticamente significativa quanto às razões de participação nos rastreios médicos em função da toma regular de medicamentos e da qualidade em saúde, pode relacionar-se com a maior percepção de saúde como "fraca/razoável" por parte dos utentes que estão sob medicação crónica.

A metodologia considerada mais adequada e que foi utilizada nesta investigação baseou-se na aplicação de um questionário aos utentes que, por ocasião, se encontravam nas diferentes unidades de saúde inseridas no estudo. Assim, uma das limitações reside no facto da amostra utilizada ser de conveniência e não aleatória, apesar de representativa da população.

Após obtenção do consentimento informado por escrito, o questionário foi preenchido pelos próprios utentes, de forma a evitar o viés do entrevistador. Contudo, a utilização do analfabetismo como critério de exclusão pode ter conduzido a uma menor representatividade de indivíduos sujeitos a maiores desigualdades sociais e habitualmente de uma faixa etária mais elevada.

De igual forma, a aplicação dos questionários pela própria investigadora, que involuntariamente acaba por eleger os utentes que interpela, pode ter sido um factor de viés. A aplicação de inquéritos com base na voluntariedade poderia ter contornado esta circunstância. No entanto, os resultados obtidos talvez fossem diferentes visto que o grupo de voluntários poderia representar uma amostra de inquiridos com melhor qualidade em saúde ou que mais frequentemente aceita os rastreios, o que conduziria a um viés de auto-selecção. 
A própria ansiedade criada pelo aguardar de uma consulta e o facto de não ter sido utilizada uma sala específica para o efeito podem também ter influenciado os resultados.

De igual modo, a aplicação do questionário exclusivamente em Unidades de Saúde pode ser um factor de confundimento uma vez que os utentes que utilizam com maior frequência estas unidades podem ser aqueles que estão mais adstritos à oferta de cuidados preventivos e que participam mais em rastreios.

Para estudos futuros, seria importante avaliar se os resultados obtidos em instituições de saúde são semeIhantes aos encontrados numa amostra populacional fora deste contexto.

\section{Referências Bibliográficas}

1. Harris R. Overview of Screening: Where Are and Where We May Be Headed. Epidemiol Rev. 2011; 33:1-6.

2. Jamoulle M, Gomes LF. Prevenção quaternária e limites em medicina. Rev Bras Med Fam Comunidade. 2014; 9(31):186-191.

3. Melo M. A prevenção quaternária contra os excessos da Medicina. Rev Port Clin Geral. 2007; 23:289-293.

4. Norman AH, Ética médica e rastreamento: em quais as evidências deveríamos nos apoiar?. Rev Bras Med Fam Comunidade. 2014; 9(31):105-107.

5. Grimer DA, Schulz KF. Uses and abuses of screening tests. The Lancet. 2002; 359: 881-884.

6. Qaseem A et al. Appropriate Use of Screening and Diagnostic Tests to Foster High-Value, Cost-Conscious Care. Ann Intern Med. 2012; 156:147-149.

7. Woolf SH, Harris R. The Harms of Screening New Attention to an Old Concern. JAMA. 2012; 307(6):565-566.

8. Hubbard RA, Miglioretti DL. A semi-parametric censoring bias model for estimating the cumulative risk of a false-positive screening test under dependente censoring. Biometrics. 2013; 69(1):245-253.

9. Wegwarth O, Gigerenzer G. Overdiagnosis and Overtreatment: Evaluation of What Physicians Tell Their Patients About Screening Harms. JAMA Internal Medicine. 2013; 173(22):2086-2087.

10. Berg CD. The Prostate, Lung, Colorectal and Ovarian Cancer Screening Trial: The prostate cancer screening results in context. Acta Oncologica. 2011; 50(1):12-17.

11. Rove KO, Craeford ED. Randomized controlled screening trials for prostate cancer using prostate-specific antigen: a tale of contrasts. World J Urol. 2012; 30:137-142.

12. Peres J. Risks of PSA Screening Now Better Understood. JNCI Oxford Journals. 2013; 105(21):1590-1592.

13. Esserman L. et al. Addressing overdiagnosis and overtreatment in cancer: a prescription for change. Lancet Oncol. 2014; 15:e234-e242.

14. Jamoulle $\mathrm{M}$ et al. Working Fields and Prevention Domains in General Practice / Family Medicine. 2002. [update:
É de notar, que existe ainda um conhecimento insuficiente sobre o impacto da promoção e educação para a saúde. A necessidade de capacitação dos utentes afigura-se ser fundamental, particularmente no conhecimento acerca dos objectivos, limitações, benefícios e riscos dos rastreios em saúde.

\section{Agradecimentos}

Aos Coordenadores e restantes profissionais de saúde das USF Viseu Cidade, USF Lusitana, USF Grão Vasco e UCSP D. Duarte pela receptividade e disponibilidade.

2002 Sept. 18; cited 2014 July 20]. Available from: http:// docpatient.net/mj/prev.html.

15. Organização Mundial de Saúde. A declaração de Jacarta sobre promoção da saúde no século XXI. 1997; 1-8. [cited 2014 July 1]. Available from: http://www.who.int/ healthpromotion/conferences/previous/jakarta/en/hpr_ jakarta_declaration_portuguese.pdf

16. Jamoulle M. Quaternary prevention: First, do not harm. 11th Congres of the Sociedade Brasileira de Medicina de Família e Comunidade. 2011; 1-11.

17. Kuehlein T, Sghedoni D, Visentin G, Gérvas J, Jamoulle M. Quaternary prevention: a task of the general practitioner. Primary Care. 2010; 1-10. [cited 2014 July 1]. Available from: http://www.primary-care.ch/docs/primarycare/archiv/ de/2010/2010-18/2010-18-368_ELPS_engl.pdf.

18. Bentzen $\mathrm{N}$ et al. WONCA dictionary of general/family practice. WONCA International Classification Committee. 2003; 1-147. [cited 2014 July 1]. Available from: http:// www.cff.org.br/userfiles/5\%20-\%20BENTZEN\%20N_\%20 Wonca\%20Dictionary\%20of\%20General\%20Family\%20 Practice.pdf.

19. Oliveira CC, Reis A. Questões epistemológicas e bioéticas da prevenção quaternária. Physis Revista de Saúde Coletiva. 2012; 22(4):1485-1502.

20. Tesser CD. Quaternary Prevention for the humanization of Primary Health Care. O Mundo da Saúde. 2012; 36(3):416-426.

21. Gérvas J, Fernández MP. Genética y prevención cuaternaria. El ejemplo de la hemocromatosis. Aten Primaria. 2003; 32(3):158-62.

22. Norman AH, Tesser CD. Prevenção Quaternária na atenção primária à saúde: uma necessidade do Sistema Único de Saúde. Cad. Saúde Pública. 2009; 25(9):2012-2020.

23. Gérvas J, Fernández MP. Uso y abuso del poder médico para definir enfermedad y factor de riesgo, en relación com la prevención cuaternaria. Gac Sanit. 2006; 20(3):66-71.

24. Martínez González C, et al. Prevención cuaternaria. La 
contención como imperativo ético. An Pediatr (Barc). 2014. [cited 2014 July 20]. Available from: http://dx.doi. org/10.1016/j.anpedi.2014.04.029.

25. Almeida LM. Da prevenção primordial à prevenção quaternária. Revista Portuguesa de Saúde Pública. 2005; 23(1):91-96.

26. Heath I. Combating Disease Mongering: Daunting but Nonetheless Essential. PLoS Medicine. 2006; 3(4):0448-0451.

27. Moynihan R, Heath I, Henry H. Selling sickness: the pharmaceutical industry and disease mongering. BMJ. 2002; 324:886-891.

28. Isaacs D. Disease Mongering. Journal of Pardiatrics and Child Health. 2013; 49: 509-510.

29. Gérvas J, Fernández MP. Uso y abuso del poder médico para definir enfermedad y factor de riesgo, en relación com la prevención cuaternaria. Gac Sanit. 2006; 20(3):66-71.

30. Norman A, Tesser C. Quaternary prevention in primary care: a necessity for the Brazilian Unified Nationl Health System. Cad. Saúde Pública. 2009; 25(9):2012-2020.

31. Gérvas J. Innovación Tecnológica en Medicina: Una Visión Crítica. Rev Port Clin Geral. 2006; 22:723-727.

32. Broeiro P, Maio V. Polifarmacoterapia: estratégias de racionalização. Rev Port Clin Geral. 2008;24:625-631.

33. Briss $P$ et al. Promoting Informed Decisions About Cancer Screening in Communities and Healthcare Systems. Am J Prev Med. 2004; 26(1):67- 80.

34. Mullen PD et al. Measures Used in Studies of Informed Decision Making About Cancer Screening: A systematic Review. Ann Behav Med. 2006; 32(3):188-201.

\section{Conflito de Interesses}

Os autores declaram não ter qualquer conflito de interesses para as matérias versadas neste trabalho.

\section{Endereço para Correspondência:}

Ana Filipa Correia de Sousa

Telemovel: 964456783

Email: anafsousa@live.com.pt

Rua Estevão Lopes Morago, lote $17,2^{\circ}$ D, Marzovelos

3510-085 Viseu
35. Health Promotion Glossary. Worth Health Organization. 1998; vi: 1-21. [cited 2014 July 1]. Available from: http:// www.who.int/healthpromotion/about/HPR\%20Glossary\%201998.pdf.

36. Brett AS, McCullough LB. Addressing Requests by Patients for Nonbeneficial Interventions. JAMA. 2012; 307(2):149-150.

37. Haggerty J et al. Patients'anxiety and expectations, How they influence family physicians' decisions to order cancer screening tests. Can Fam Physician. 2005; 51:1658-1659.

38. Antoniou SA et al. Reflections of the hippocratic oath in modern medicine. World J Surg. 2010; 34:3075-3079.

39. Nguyen M, Larocque D, Paquete D. Quebec breast cancer screening program. A study of the perceptions of physicians in Laval, Que. Canadian Family Physician. 2009; 55:614-20.

40. Cooper CP, Gelb CA, Hawkins NA. How many "Get Screened" messages does it take? Evidence from colorectal cancer screening promotion in the United States. Elsevier Inc. Preventive Medicine. 2012; 60:27-32.

41. Ogden, J. Health Psychology. A Textbook. Buckingham: Open University Press. 1999.

42. Gomes R, Nascimento E, Araújo F. Por que os homens buscam menos os serviços de saúde do que as mulheres? As explicações de homens com baixa escolaridade e homens com ensino superior. Cad. Saúde Pública. 2007; 23(3):565-574. 